Revue musicale OICRM

\title{
L'invention musicale face à la nature dans Grizzly Man, The Wild Blue Yonder et La grotte des rêves perdus de Werner Herzog
}

\section{Vincent Deville}

Volume 5, numéro 1, 2018

Ce que le cinéma nous apprend de la musique

URI : https://id.erudit.org/iderudit/1044446ar

DOI : https://doi.org/10.7202/1044446ar

Aller au sommaire du numéro

Éditeur(s)

Observatoire interdisciplinaire de création et recherche en musique (OICRM)

ISSN

2368-7061 (numérique)

Découvrir la revue

Citer cet article

Deville, V. (2018). L'invention musicale face à la nature dans Grizzly Man, The Wild Blue Yonder et La grotte des rêves perdus de Werner Herzog. Revue musicale OICRM, 5(1), 103-120. https://doi.org/10.7202/1044446ar

\section{Résumé de l'article}

Quand le cinéaste allemand Werner Herzog réalise ou produit trois documentaires sur la création et l'enregistrement de la musique de trois de ses films entre 2005 et 2011, il effectue un double geste de révélation et d'incarnation : les notes nous deviennent visibles à travers le corps des musiciens. Ce faisant, Herzog nous délivre son art poétique : les formes musicales répondent à la nature, si présente dans ses films ; la nature est envisagée comme un instrument d'accord tandis que la musique devient une puissance d'estrangement, de désaccord de nos perceptions ; et en nous donnant accès aussi bien à un infigurable - l'être moral - qu'au corps matériel fait de chair et d'os, la musique nous ouvre la voie qui mène au coeur du monde. 


\title{
L'invention musicale face à la nature dans Grizzly Man, The Wild Blue Yonder et La grotte des rêves perdus de Werner Herzog
}

\author{
Vincent Deville
}

\section{Résumé}

Quand le cinéaste allemand Werner Herzog réalise ou produit trois documentaires sur la création et l'enregistrement de la musique de trois de ses films entre 2005 et 2011, il effectue un double geste de révélation et d'incarnation : les notes nous deviennent visibles à travers le corps des musiciens. Ce faisant, Herzog nous délivre son art poétique : les formes musicales répondent à la nature, si présente dans ses films ; la nature est envisagée comme un instrument d'accord tandis que la musique devient une puissance d'estrangement, de désaccord de nos perceptions ; et en nous donnant accès aussi bien à un infigurable - l'être moral - qu'au corps matériel fait de chair et d'os, la musique nous ouvre la voie qui mène au cœur du monde.

Mots clés : Herzog ; Grizzly Man ; nature ; mimèsis ; incarnation.

\begin{abstract}
When German filmmaker Werner Herzog directs or produces three documentaries on the creation and recording of the music of three of his films between 2005 and 2011, he performs a double gesture of revelation and incarnation: the notes become visible through the body of the musicians. In doing so, Herzog reveals his poetic art: musical forms respond to nature, so present in his films; nature is seen as an instrument of harmony while music becomes a power of estrangement, getting our perceptions out of tune; and by giving us access to both the infigurable - the moral being - and the material body made of flesh and bone, the music opens a path to the heart of the world.
\end{abstract}

Keywords: Herzog; Grizzly Man; nature; mimesis; incarnation. 
I said to myself: "Why shouldn't I try at least once in my life to transform everything into music, every action, every word into music." I have worked in a similar way, to a certain degree, in my films, transforming an image that is physically there into something more intensified, into something elevated or stylized (Herzog [1985] 2014a, p. 97).

«A VERY MUSICAL PERSON »

Le cinéaste allemand Werner Herzog, s'il s'est toujours défendu d'être un érudit ou un savant, n'en est pas moins un amateur éclairé. Ainsi son rapport à la musique, éclectique et marqué d'une insatiable curiosité, qui traverse et nourrit l'ensemble de sa filmographie. On y trouve en effet, dans le désordre et selon la plus grande liberté d'agencements :

- des musiques traditionnelles et populaires, glanées au hasard des tournages au quatre coins du monde, comme un duo de lyre crétoise et de bouzouki dans Derniers mots (1968) ; des folk songs de Leonard Cohen dans Fata Morgana (1971) ; du didgeridoo dans Le pays où rêvent les fourmis vertes (1984); des chants diphoniques mongols dans Les cloches des profondeurs (1993);

- des tubes classiques, du Canon de Pachelbel au Concerto pour piano $n^{\circ} 3$ de Beethoven, de Peer Gynt de Grieg au Deuxième trio pour piano, violon et violoncelle de Schubert, et Wagner de manière récurrente ${ }^{1}$;

- des compositeurs moins connus du grand public et à la production singulière, comme le compositeur italien de la Renaissance Carlo Gesualdo, à qui Herzog consacre un portrait fantasque : Gesualdo, mort à cinq voix (1995) ;

- des morceaux joués en direct par les protagonistes des films, et que l'on pourrait qualifier de musique brute comme l'on parle d'art brut : Bruno S. en pianiste dissonant dans L'énigme de Kaspar Hauser (1974) ou en homme-orchestre dans La ballade de Bruno (1977), film dans lequel on rencontrera également deux proxénètes aux allures barbares vandaliser un piano, ou encore une poule pianiste et un canard percussionniste ;

- des collaborations fortes avec des compositeurs : Florian Fricke alias Popol Vuh - Aguirre, la colère de Dieu (1972) ; La grande extase du sculpteur sur bois Steiner (1974); Cour de verre (1976); Nosferatu, fantôme de la nuit (1979); Fitzcarraldo (1982) ; Gasherbrum, la montagne lumineuse (1985) ; Cobra Verde (1987) - ou plus récemment le violoncelliste expérimentateur Ernst Reijseger The White Diamond (2004) ; The Wild Blue Yonder (2005) ; Dans l'oeil d'un tueur (2009); La grotte des rêves perdus (2010); Salt and Fire (2016).

Ce qui frappe, lorsqu'on passe d'un film à l'autre au sein de cette vaste filmographie rassemblant 68 projets (en 2017), tous types de production et de format confondus, c'est la plasticité avec laquelle les musiques migrent d'un univers à un autre, se retrouvent de film en film, ne restant jamais cantonnées à un espace supposé d'origine, et contribuant ainsi à renforcer l'idée d'un grand œuvre herzogien. 
Bien qu'il déclare avoir échoué à apprendre à jouer de la flûte avec sa mère, et être l'un des rares metteurs en scène d'opéra ${ }^{2}$ à ne pas savoir lire la musique, Herzog se définit lui-même comme une " personne très musicale ${ }^{3}$ " (Herzog 2014b, p. 304). Pour autant, on peut se demander s'il n'a pas de réelles velléités de musicien, comme peuvent le suggérer trois documentaires qui décrivent la production de musiques originales pour trois de ses films :

- In the Edges, The Grizzly Man Sessions(2005) pour la musique de Grizzly Man(2005), dont les sous-titres en ouverture annoncent un film " with Richard Thompson and Werner Herzog ", contribuant à placer à un même niveau le musicien, compositeur et leader du groupe constitué pour l'occasion, et le cinéaste, ce que confirmera un plan à la fin du film, les cadrant ensembles, comme s'ils étaient tous deux compositeurs de la musique. Le film, réalisé par Erik Nelson, n'est ni signé ni produit par Herzog, il est cependant monté par Joe Bini, son monteur attitré depuis Petit Dieter doit voler (1997).

- Le titre Requiem in Space. Werner and Ernst Make Music (2005) insiste littéralement sur le fait que Werner Herzog et le violoncelliste Ernst Reijseger forment un duo de musiciens pour créer la musique de The Wild Blue Yonder (2005). Le générique ne crédite pas de réalisateur, mais un directeur du montage, Nicolas McClintock ; André et Nicholas Singer à la production ; et le film est coproduit par la société Werner Herzog Filmproduktion.

- Ode to the Dawn of Man (2011) pour la musique de La grotte des rêves perdus est cette fois réalisé par Herzog, qui assure lui-même l'image et le son, ayant improvisé ce tournage au débotté, à l'aide d'une petite caméra non professionnelle prêtée par une personne présente au moment de l'enregistrement, et qui lui permet de se tenir au plus près d'Ernst Reijseger, là encore comme d'égal à égal au cour du processus de création ${ }^{4}$.

Comme cette déclaration lyrique le suggère : "I can thank God on my knees that cinema is probably the closest to music that you can find. Cinema is not related that much to literature, it is not that much related to other art forms like painting. It's very very close to music » (Herzog 2005a), il y a fort à parier qu'Herzog ait en effet trouvé dans le cinéma un dérivatif à la musique, et qu'il ait conduit sa carrière de cinéaste avec l'esprit d'un musicien. Il parle d'ailleurs très tôt de ses prédispositions musicales exprimées à travers le cinéma :

Je suis très ébranlé, très agité par la musique, presque autant que par les films. Parfois, la véritable qualité d'une image ne peut être appréhendée que par la musique. Un exemple serait le travelling sur les dunes de sable dans Fata Morgana. Après l'avoir

2 Herzog a en effet mis en scène une douzaine d'opéras, montés sur des scènes internationales entre 1986 et 2013.

3 «I'm a very musical person».

4 Filmé lors des deux journées d'enregistrement, en juillet 2010 dans une église protestante de Harlem, Hollande, le film sera achevé en avril 2011. 
regardé plusieurs fois à la Moviola, je me suis rendu compte d'un seul coup que c'était un paysage féminin. J'ai alors accompagné ces paysages par un chœur de femmes extrait de La Messe du couronnement de Mozart et les spectateurs ont pu alors en saisir la qualité féminine avec beaucoup de netteté (Herzog 1975, p. 12).

Chez Herzog, musiques et images sont intimement liées entre elles, ainsi qu'au processus de création lui-même. Dans Requiem in Space, le cinéaste déclare : "Cela peut paraitre curieux, mais pour The Wild Blue Yonder, et parfois pour d'autres films, j'avais de la musique en tête, et la totalité du film, pas seulement la musique » (Herzog 2005b).

Dès lors, ne faudrait-il pas envisager les documentaires sur la fabrication de la musique des films de Werner Herzog comme de véritables making of des films eux-mêmes ? Ces objets filmiques impurs, réalisés sans grands moyens, a priori annexes et à destination de bonus DVD, tout en nous donnant à mieux comprendre et à entendre la musique des films, ne nous donnent-ils pas aussi à mieux saisir les enjeux des films pour lesquels ces musiques ont été conçues ? Est-ce par ailleurs un hasard si ces trois making of concernent des musiques qui reposent en partie sur de l'improvisation et si le sujet des trois films touche à la question métaphysique de la place de l'homme dans la nature ? Nous verrons en effet que par sa puissance de révélation d'un infigurable et par sa puissance de figuration de l'être moral, la musique chez Herzog concerne tout d'abord la mimèsis. Pour découvrir ensuite une nature entendue comme instrument d'accord d'une part, puis une musique conçue comme puissance d'estrangement d'autre part. Et pour comprendre enfin comment, par-delà la mort, à travers les questions de création et d'incarnation, la musique est une condition essentielle d'accès au corps.

IMITATIONS DE LA NATURE

Pour la musique de Grizzly Man, Herzog met en place un processus de création original. Il réunit dans un studio d'enregistrement, deux jours de décembre 2004, cinq musiciens ${ }^{5}$ qui n'ont jamais joué ensemble et qui vont se livrer à une série d'improvisations, sous le regard et l'écoute du cinéaste. Dans In the Edges, Herzog déclare qu'il a une idée très précise de ce qu'il veut et qu'il sait identifier les moments musicaux qui lui conviennent. Ainsi, après avoir demandé aux musiciens de reprendre leur introduction, parce qu'il n'a pas « reconnu » la première phrase musicale, il dit de Richard Thompson : "I think he just got it » (Herzog 2005a), comme si Herzog cherchait à capturer quelque chose et qu'il avait dans un second temps reconnu cette chose. Sa recherche semble concerner une dimension brute ( $\mathrm{raw}$ ), qui se révèle assez rapidement dans les propos de Herzog consister à retrouver dans la musique la part sauvage de la nature, omniprésente dans Grizzly Man: "I need the raw soul of you. Because what we have in the film has such a raw quality. The music has to be of the same quality" (ibid.).

5 Richard Thompson, Danielle DeGruttola, John Hanes, Jim O’Rourke, Damon Smith, auxquels il faut ajouter le producteur Henri Kaiser. 
Ce que corrobore le guitariste Richard Thompson, en quête d'une musique qui n'arrondirait pas ses angles, et qui donnera son titre au documentaire :

If you repeat music too often, if you rub the edges of music, you really take away the music itself, the music is in the edges, it's in the rough place. If you smooth it over, there's really nothing left, you would have lots of notes left but there is no music. So it's always a striving to keep something alive, something fresh, something that adds a vitality to it (ibid.).

Il y aurait donc deux types de musique, une musique rugueuse, dure (rough) ou encore brute, crue (raw), opposée à une musique assouplie, polie ${ }^{6}$. Pour Richard Thompson, radical sur la question, il y aurait en fait tout bonnement une musique qui serait de la musique et une autre qui n'en serait pas. Ce que l'on comprend alors, c'est l'affinité profonde entre cette conception d'une musique brute et la sauvagerie de la nature, telle que Herzog veut la démontrer dans le film, en opposition à une vision édulcorée, «à la Disney ", telle qu'un Timothy Treadwell, le personnage central de Grizzly Man, pourtant lui-même largement confronté dans ses expéditions aux conditions rudes de la vie en pleine nature, a pu les véhiculer auprès de son public. Herzog parle également d'une musique qui doit d'emblée faire autorité, donnant à sentir que nous sommes, comme Timothy Treadwell dans le film, face à la loi de la nature ("This is the law of the Earth»). En somme, Herzog parle ici d'une musique dotée de qualités mimétiques, comme lorsqu'il insiste auprès des musiciens : "Avec le bruit du vent, et les interférences dans le micro de Treadwell, le film a un aspect réaliste qu'il faut conserver. La musique doit être du même acabit » (ibid.).

Dans son ouvrage La nature et la musique, Emmanuel Reibel revient sur la nature comme source d'inspiration majeure pour les artistes, expliquant que l'homme a toujours renchéri sur les manifestations sonores de la nature, par exemple lorsqu'il souffle dans des roseaux taillés, percute des peaux et carapaces ou taille des arbres pour construire des instruments : "À défaut de pouvoir facilement imiter la nature, la musique souhaite souvent lui faire écho, afin d'entrer en résonance avec elle » (Reibel 2016, p. 9-10). On touche là à une dimension anthropologique, au besoin inné de l'homme de faire de la musique, de fabriquer des instruments avec ce qui lui tombe sous la main, morceaux de bois, d'os (les flûtes dans La grotte des rêves perdus) ou de plastique selon les époques, ainsi que Werner Herzog en fait l'expérience sur le tournage de Fitzcarraldo:

Quelqu'un a joué de la flûte dans le camp, cette nuit, dans un silence de mort. J'ai fini par me lever, à minuit, et j'ai vu un des aides de cuisine jouant avec le tube en plastique d'un conduit d'eau, qu'il avait percé. La nuit était si exceptionnellement calme que je l'ai sévèrement rabroué et que je me suis relevé plus tard dans la nuit pour m'assurer que le fleuve était toujours là (Herzog 2008a, p. 289).

6 Dans le même esprit, s'inspirant de Claude Lévi-Strauss (Lévi-Strauss 1964), le critique de cinéma Serge Daney travaillera l'opposition cru/cuit (Daney 1981) afin de revendiquer et valoriser un cinéma du cru (Jean Renoir, Robert Bresson, Jean-Luc Godard...) au détriment d'un cinéma du cuit (Bertrand Tavernier, Claude Berri...). 
Reibel poursuit en évoquant les incantations qui cherchent à conjurer les forces du vent, du feu, de la pluie :

Par les mystères de la musique, il serait possible de communier avec la nature, voire d'agir sur elle. [...] Si Orphée est devenu le modèle mythique de tous les poètes et musiciens, c'est parce que la nature lui obéit : accompagné à la lyre, son chant était capable, dit-on, de dompter les animaux les plus sauvages, de retenir le cours des ruisseaux, de commander aux éléments, voire de triompher de la mort (Reibel 2016, p. 10-13).

C'est là une description de Timothy Treadwell et du programme qu'on le voit accomplir dans Grizzly Man : dompter les ours de sa voix enfantine, éraillée et haut perchée ; modifier le cours des ruisseaux pour libérer le passage des saumons ; improviser, de rage, un chant de la pluie blasphématoire pour faire enfin pleuvoir ; réaliser un film voué à lui survivre.

Herzog donne dans In the Edges une description de la musique de Grizzly Man, qui serait selon lui simplement composée d' « un motif récurrent du début à la fin du film, et un moment décisif au milieu »(Herzog 2005a). Quel est ce moment précis, que Herzog n'explicite pas, et qu'il nous revient donc d'identifier ? La musique du film, emmenée par la guitare de Richard Thompson, développe globalement des thèmes d'harmonie entre Treadwell et la nature, positifs, majestueux. Une première étape introduit une réserve : ce sont les notes sombres qui apparaissent dans la partition, jouées au violoncelle et non plus à la guitare, qui descendent dans le grave au fur et à mesure que Treadwell descend une pente et entre dans l'eau pour se baigner avec un ours, alors que la voix over nous explique que Treadwell a franchi une barrière de plus de 7000 années, qui séparait les hommes des ours sauvages. Une seconde étape vient accomplir le moment décisif. D'une part, elle se produit à l'exact centre du film, comme le suggère Herzog (" au milieu »), juste après la séquence où le cinéaste écoute au casque les derniers instants de Treadwell. D'autre part, la nature et la fonction même de la musique ont radicalement changé. Le violoncelle a encore gagné en graves, par l'adjonction d'une contrebasse, les deux instruments cherchant l'un et l'autre, très mimétiquement, à redoubler la lutte et les grognements des ours en train de se battre. La musique reproduit littéralement un phénomène naturel, brut, sans plus s'encombrer de règles musicales savantes. De l'ouverture mélodique au moment proprement mimétique, le monde a été transformé en musique, la musique en monde : musique et nature deviennent indiscernables.

La scène qui suit le combat des ours travaille une forte opposition au moins à deux titres : visuellement, on est passé de la fange, où luttaient les ours, à la pureté blanche et bleutée des glaciers ; musicalement, nous sommes revenus à la lead guitar de Thompson, qui pose à nouveau l'autorité de la nature. Or, c'est bien encore d'un moment mimétique dont il s'agit là, tandis que Herzog décrit le glacier crevassé comme une métaphore de l'âme fissurée de Treadwell. Une légère pause dans la voix over et un mixage qui augmente le volume après que Herzog a prononcé la sentence : "Il me semble que ce paysage bouleversé est une métaphore de son âme " ne laisse aucun doute : les vibrations et distorsions des cordes de la guitare électrique ne sont pas moins une métaphore de la fêlure psychologique de Treadwell. Herzog avait déjà tenté un tel rapprochement dans Gasherbrum, la montagne lumineuse (1985), filmant en panoramique 
les crêtes escarpées et dentelées d'une chaîne de montagne, s'interrogeant en voix over sur les exploits extrêmes de l'alpiniste Reinhold Messner. C'est là aussi au moment même où dans ce film il prononce la phrase : "Ces monts et ces sommets ne sont-ils pas une qualité enfouie au plus profond de nous ? ", que sur la bande-son se déclenchent les nappes vocales de Popol Vuh. Cette association entre panoramique, voix over et musique donne à celle-ci une qualité introspective, une puissance de révélation d'un infigurable, en esquissant le portrait moral d'un être. Face aux paysages, face à la musique, Herzog se pose en artiste visionnaire, au sens de personne douée d'une vision, qui prévoit ses images et ses musiques, mais qui sait aussi les reconnaître quand il est en leur présence : tel paysage, telle ligne mélodique qui proprement rencontrera ou non son projet artistique. Nous pouvons nous-mêmes reconnaître dans ce rapport à la création la dimension platonicienne de reconnaissance comme réminiscence ${ }^{7}$, qui postule une immortalité de l'âme, laquelle aurait appris antérieurement ce qui est reconnu, ressouvenu dans sa forme incarnée ici et maintenant.

Si nous repensons maintenant à Herzog auprès de ses musiciens, les guidant sur le chemin de la création, jusqu'à identifier et reconnaître ce qu'il cherche, nous sommes bien en présence d'une réminiscence platonicienne, qui donne accès ici à la nature première qui nous a échappé, comme le suggère le philosophe Hans Blumenberg :

une certaine présence du toujours-déjà-là s'introduit subrepticement dans ce qu'il [l'homme] a créé, comme s'il s'agissait là d'un produit de la simple nature. [...] dans la marge de manœuvre investie par la création libre, se cristallisent subrepticement des structures dans lesquelles on peut reconnaître, sous une forme qui lui confère une force de conviction nouvelle, l'archaïsme, l'être-depuis-toujours d'un fond originel de la nature (Blumenberg [1981] 2010, p. 88-89).

La " création libre ", voilà bien un terme pour désigner l'approche artistique de Werner Herzog : laisser les sujets de films venir à lui ; s'adapter à tous types de production et de format selon les lieux et les époques; faire appel à des musiciens improvisateurs et créer des combinaisons musicales inédites ; créer des conditions de production qui ouvrent la création à la contingence. Ce que Blumenberg résumera ainsi, citant Paul Klee : "Voilà en fin de compte ce qui était en jeu, "l'essentialisation du contingent" "(ibid.).

\section{LA NATURE COMME INSTRUMENT D'ACCORD}

Dans Grizzly Man, Herzog manifeste son admiration à l'égard de Treadwell quand celui-ci laisse sa caméra tourner et qu'il enregistre l'arrivée soudaine dans le plan d'un renard et d'un renardeau. Herzog, louant la magie inexplicable du cinéma, déclare en voix over : "Il a enregistré des moments d'improvisation fantastiques » (Herzog 2005c). Allant même jusqu'à en faire un modèle de production à revendiquer : «Il a réalisé des images telles que les équipes de studio, avec leurs règles syndicales, ne 
pourront jamais en réaliser » (ibid. $)^{8}$. Ce qui n'étonne pas, de la part de Herzog qui a toujours aimé déclarer que faire des films n'était pas un travail, et ceci à propos d'un Treadwell qui préférait à l'American Way of Life le mode de vie franciscain d'un apôtre de la nature. Cette conception du cinéma basée sur la contingence comme force de proposition pour la mise en scène peut faire écho à la conception de la nature du philosophe et poète Novalis :

Pour comprendre la Nature, on doit la laisser, intérieurement, se former selon sa propre logique, se développer en toute son intégrité. On doit, dans cette entreprise, ne se laisser déterminer que par le grand désir, la divine aspiration vers ces êtres qui nous ressemblent, et par les conditions qui nous sont nécessaires pour les entendre ; car la Nature tout entière ne se peut concevoir et comprendre que comme instrument d'accord et moyen d'intelligence, entre eux, pour des êtres doués de raison (Novalis [1802] 1975, p. 59, nous soulignons).

C'est la grande aspiration de Treadwell vers les animaux sauvages, de Herzog vers Treadwell, ou encore de Herzog vers des musiciens aux origines des plus variées ${ }^{9}$. Dans les collaborations musicales inédites qu'il provoque, il s'agira alors pour les musiciens aux styles et parcours hétérogènes de déceler une forme d'accord selon le principe niché au cœur des films : trouver une forme de cohésion et d'harmonie en envisageant la nature comme instrument d'accord primordial, pour reproduire et produire in fine quelque chose de sa force, de sa rudesse, de sa brutalité.

Dans les procédés de création qu'il met en œuvre, Herzog retrouve une conception de la nature créatrice, ainsi que l'expose Marcel Conche : "Pourquoi la Nature ne pourrait-elle varier ses méthodes, ses procédés, ses trouvailles, ses gestes, comme un artiste?"(Conche 2001, p. 6). Dans sa grande attention aux phénomènes naturels, on sent chez Herzog non seulement une volonté mais une capacité et une affinité à intégrer dans l'œuvre ce qui se présente à lui et qu'il sait reconnaître. Ainsi, sur le tournage de Fitzcarraldo: "Je me suis réveillé à trois heures du matin, hier ; la lune éclairait une brume blême sur les pentes. Il m'a semblé évident que je devais filmer ce moment insaisissable. » (Herzog 2008a, p. 313). C'est la même logique qui préside au tournage du Cuirassé Potemkine (1925). Alors que des brumes se lèvent, Eisenstein dira qu'elles s'accordent à l'émotion des funérailles et s'invitent dans le film. Le cinéaste multiplie le vocabulaire musical pour parler de la conception du film, et finira par nommer ce passage, une fois monté, la « symphonie des brouillards ${ }^{10}$ ».

8 Herzog poursuit sur ce registre dans un entretien : "The film shows these wonderful moments, and I want to give him the space and the credit, as a fellow filmmaker that all of us can only envy. Even if I gave you $\$ 50$ million of Hollywood money, you could never achieve what he did with a little video camera. And I believe the best of the best is in this film " (Herzog [2005] 2014a, p. 155-159).

9 Pour The Wild Blue Yonder, Herzog réunit le chanteur sénégalais de langue wolof Mola Sylla, les chanteurs polyphoniques sardes du groupe Tenore e Cuncordu de Orosei, sous la houlette du violoncelliste hollandais Ernst Reijseger.

10 «Il faut assez de rigueur pour savoir exactement la qualité de la sonorité qu'on désire donner et assez d'indépendance pour accueillir les possibilités qui se présentent à l'improviste et qui sont susceptibles de créer cette sonorité. [...] Le hasard oblige à des solutions tranchantes. La clé musicale devant rester la même, 
En filigrane, ce dont il est question pour Eisenstein ou Herzog, c'est de maintenir de la vie dans le film, de garder le film vivant : "Vous vivez votre vie et vous restez ouvert aux occasions et à tout ce que vous voyez. [...] Je reste ouvert, je change, je vis et la vie reste dans mes films »(Herzog 2008b, p. 52). Herzog explique à ce propos que lorsqu'il s'est fait aider par des étudiants pour dérusher la centaine d'heures tournées par Treadwell, il a dû intervenir pour que ses jeunes collaborateurs conservent des plans qu'ils jugeaient inutiles : par exemple des plans d'herbes agitées par le vent lorsque Treadwell est sorti du cadre. Pour insister sur ce moment de vie qui a intégré son film, Herzog accumule plusieurs procédés : laisser durer ce plan vide en le sauvant de la poubelle du montage ; ajouter un bruit de vent mais non direct, puisque les pas de Treadwell qui fait retour dans le plan sont inaudibles, donnant toute sa place à la seule nature, totalement indifférente à l'humain ; cette indifférence voire discordance de la nature est redoublée par une musique dissonante exécutée aux cordes ; et, si cela n'était pas suffisant dans la forme, Herzog le formule explicitement en voix over: "Les images développent parfois leur propre vie, leur propre éclat mystérieux » (Herzog 2005c).

\section{LA MUSIQUE COMME PUISSANCE D'ESTRANGEMENT}

Une transition, dans Grizzly Man, lie le moment où Jewel parle de sa relation à Timothy en termes d'âme sœur (kindred spirit) et une séquence où l'on voit Timothy et la renarde Spirit dans une relation de grande complicité, en forme d'accord. On assiste à l'un des moments les plus touchants du film, dont l'émotion n'est pas étrangère à la musique. Timothy commence à parler au renard, de sa voix douce mais aussi perchée, enfantine. Des plans édéniques montrent Spirit dans des paysages de nature fabuleuse (paysage marin ensoleillé, graminées dans le vent, traversée d'une rivière...). La musique se développe en un duo guitare-piano, mais un piano qui pourrait faire penser à un jouet, au son fluet, fragile, désaccordé. Le documentaire sur la musique nous révèle que pour ce passage Jim O'Rourke joue sur un piano préparé, travaillant minutieusement à désaccorder l'instrument, à lui donner de nouvelles vibrations. Cette musique renvoie alors au paradis perdu d'une enfance, celle de Timothy, fissurée, blessée, elle-même désaccordée. Et quand Timothy, au bord des larmes, lance un message désespéré pour que les hommes cessent de tuer et torturer les renards, il évoque leur douceur tout en caressant Spirit. La musique prend en charge cette douceur, qui est aussi douce-amère.

C'est bien sûr une des fonctions de l'agencement entre des images et des notes que de créer autre chose que de l'accord ou de l'empathie : de la distance. "Pour Aguirre la musique fut composée spécialement : je voulais un chœur qui soit hors de ce monde, comme, lorsque j'étais enfant, je pensais, me promenant la nuit, que les étoiles chantaient» (Herzog 1975, p. 12).

l'accident entre alors dans la chair du film selon une loi imprescriptible. [...] la sonorité émotionnelle s'est accordée parfaitement à la conception initiale de la scène funèbre et voici que les brouillards inattendus pénétraient au cœur même du projet » (Eisenstein [1945] 1952, p. 18-29). 
À des années d'écart, Herzog, s'interrogeant sur la dénomination adéquate pour la musique de The Wild Blue Yonder, poursuit la métaphore d'une musique extraterrestre, qui dépasse les limites de notre univers : "Symphonie visuelle, Oratorio dans l'espace, Requiem For The Outter Fringes of Space... Je ne sais pas quel nom lui donner, mais cela a davantage à voir avec une forme musicale qu'une forme cinématographique. » La musique interroge les images comme un regard extérieur et distant posé sur l'humanité, à la manière du regard extra-terrestre posé par Brad Dourif dans The Wild Blue Yonder qui, avec Fata Morgana et Lessons of Darkness, s'inscrit dans un même projet : voir notre monde comme le ferait un individu arrivant de l'espace, qui ne connaitrait rien de notre civilisation (Prager 2007, p. 118). Soit exactement le principe de l'estrangement théorisé par Viktor Chklovski et repris comme méthode historienne par Carlo Ginzburg.

D'emblée, l'argument premier du concept d'estrangement résonne assez bien chez Herzog avec la quête de visions, qui passe chez lui par une opposition entre facts and truth, soit la construction d'une vérité extatique qui ne se résume pas à l'addition de faits comptables :

La fin de l'art est de nous procurer une sensation de la chose, mais une sensation qui soit une vision, et non pas seulement une reconnaissance. Pour parvenir à ce résultat, l'art utilise deux procédés : l'estrangement des choses et la complication des formes, par laquelle il cherche à rendre la perception plus ardue et à en prolonger la durée. En art, la perception est une fin en soi et doit être amplifiée. L'art est le moyen de voir quelque chose devenir ; ce qui a réellement été n'a aucune importance (Ginzburg [1998] 2001, p. 16).

Pour Ginzburg, procéder selon la méthode de l'estrangement pour voir le monde tel qu'il est vraiment, consiste encore à se défaire des postulats qui semblaient évidents pour envisager les phénomènes comme s'ils étaient dénués de sens, comme des devinettes. C'est-à-dire adopter une attitude naïve à l'égard du monde : " Comprendre moins, être ingénu, rester stupéfait sont des réactions qui peuvent nous aider à voir davantage, à saisir une réalité plus profonde, plus naturelle » (ibid., p. 26). En ce sens, un certain nombre de personnages herzogiens "naifs " pratiquent la technique de l'estrangement, sans le savoir. Mais ce sont aussi beaucoup les musiques singulières placées par Herzog sur ses images qui contribuent à l'estrangement de celles-ci et à déplacer la perception que nous en avons. Dans la célèbre scène de Signes de vie où Stroszek découvre la vallée aux mille moulins à vent, c'est bien au jeu des devinettes sonores que nous invite Herzog, plaçant sur la bande son des applaudissements ralentis mixés au chant des anges, ces bruits du vent dans les fils électriques.

Avant même sa collaboration avec Florian Fricke (qui apparaît cependant en pianiste dans le film, pour révéler à Stroszek le caractère fondamentalement méchant de la musique de Chopin), Herzog a dès son premier long métrage l'intuition d'une expérimentation sonore et musicale qui donne accès à d'autres dimensions, comme il le dira à propos du travail ultérieur de Florian Fricke :

Florian never failed to create music that has forever given us an entrée into otherwise inaccessible dimensions. [...] Florian's compositions add dimensions to a film that we never knew existed and enable us to shift our perception; they make visible what would otherwise 
remain mysterious and forever hidden in my films, and also what lies buried in our souls (Herzog 2014b, p. 302-303).

Cette puissance d'estrangement de la musique, sa capacité à désaccorder nos perceptions les plus solidement ancrées, Herzog la met en scène dans son hommage au compositeur Gesualdo, Mort à cinq voix, en faisant intervenir deux musiciens et historiens de la musique qui entretiennent chacun un rapport singulier à l'œuvre de Gesualdo et à la musique en général. Tandis que le musicologue anglais est du côté d'une approche historique, objective et chronologique de la vie du compositeur, racontée sereinement dans un intérieur calme, livres anciens et notes manuscrites à l'appui, signes du sérieux et de la scientificité d'une approche rationnelle, le musicologue américain, lui, est placé par Herzog en extérieur, sur fond de fête médiévale agitée et bruyante, racontant de manière exaltée et subjective sa découverte de l'œuvre de Gesualdo, une musique difficile qu'il faut affronter et conquérir (tandis que dans son dos détalent des chevaliers lancés sur leur monture), une musique qui nécessite de prendre des risques (comme il semble en prendre lui-même, placé si près des cavaliers au galop qui le frôlent). On pourrait presque dire que Herzog oppose ici deux rapports à la musique, l'un désincarné, l'autre très incarné. Comme le remarque Brad Prager, c'est l'ensemble de ce film qui est structuré sur un déséquilibre et un désaccord, opposant des moments de performance musicale stable à des aventures rhapsodiques et imaginaires :

Viewed in this way, the film can be read as homage to late Mannerist madrigal composition, with its alternating tonal homophonic voicing and chromatic, polyphonic rampages. Death for Five Voices, in other words, is structured in a way closer to musical form than it is to a filmic or literary narrative: an episodic, bitty and sectional structure, moreover, akin to that of Gesualdo's late madrigals (Rogers 2012, p. 204).

Et tout comme pour Timothy Treadwell la musique se fait plus sombre et dissonante à mesure que sa mort approche et que Herzog nous révèle les fissures profondes de son être, les madrigaux de Gesualdo se font eux aussi plus sombres et difficiles tandis que la folie et la mort gagnent le personnage.

\section{PAR-DELÀ LA MORT : CRÉATION ET INCARNATION}

Alité à la suite d'un attentat sur sa personne, Kaspar Hauser expose sa vision d'une foule gravissant une montagne : "Il y avait beaucoup de brouillard. Je ne voyais pas clairement. Et en haut il y avait la Mort »(Herzog 1974). Le Canon de Pachelbel accompagne majestueusement cette vision, qui se poursuit en réintégrant Kaspar pensif dans la nature. À la fin de cette séquence, comme le jeune Mozart exposé à la foire des curiosités entrevu plus tôt dans le film, Kaspar semble chercher un trou noir au fond d'une cuve remplie d'eau. Alors qu'il brouille la surface de l'eau avec sa main, faisant ainsi disparaître le reflet de son visage, les bruits de nature, d'oiseaux et de mouches vrombissantes s'intensifient. On retrouve Kaspar à l'intérieur devant son piano, s'exerçant laborieusement, buttant toujours sur les rythmes et les notes. Et là encore, juste avant de retrouver Kaspar atteint d'une blessure qui lui sera fatale, la musique est recouverte par le chant des oiseaux à la faveur d'un raccord sur le jardin. Musique, mort et nature intimement liées, la nature agissant comme chant qui reprend 
ses droits sur la musique savante des hommes. Et comme le montre Marcel Conche, si la nature est du côté de la vie, elle est tout autant du côté de la mort :

" Il n'est rien de sûr, dit Critias, sinon que mourir est le lot de celui qui est né ». Est-ce la seule chose toute à fait certaine ? Peut-être pas. Mais il n'est rien de plus certain. Or, l'évidence de la mort est évidence de la Nature : car la Nature est insistante en nous par la mort. [...] Dire que l'étude de la Nature porte « sur la génération et la destruction » des êtres, c'est dire qu'elle a pour objet, pour tout ce qui est, la venue à être et la cessation d'être : la vie et la mort. Penser la Nature comme phusis, c'est, suivant l'étymologie, penser la Source génératrice, l'origine et la naissance, la croissance ; mais c'est aussi penser le déclin et la mort. L'évidence de la Nature et l'évidence de la mort ne sont qu'une seule et même évidence (Conche 2001, p. 12).

Comme nous venons de le voir avec Kaspar, pour Werner Herzog, la représentation de la Nature et de la mort ne sont qu'un seul et même problème figuratif, aux implications musicales fortes. C'est ainsi une même question que posent Gesualdo, Grizzly Man, The Wild Blue Yonder ou La Grotte des rêves perdus : comment figurer l'humain, comment figurer le corps mort, comment figurer le premier ou le dernier des hommes ? Comment figurer dans des portraits in absentia le corps de Timothy déchiqueté par un ours, celui de Gesualdo introuvable et pourtant partout présent dans le film, ou encore celui de l'être humain disparu d'une planète moribonde ? En se confrontant au problème de l'incarnation.

Très tôt dans l'œuvre, dès les premiers courts et le premier long métrage, la musique ouvre littéralement sur le corps. Ce à quoi la musique donne accès, on pourrait dire que c'est le corps. Dans Derniers mots, une série de témoins évoque un personnage récalcitrant, un joueur de lyre qui vit en marge sur une île. Alors que son retour parmi la société est évoqué, un raccord fait apparaître un joueur de lyre, accompagné d'un joueur de bouzouki, emporté dans un duo d'improvisation animé. Comme si après les nombreuses paroles à son propos, il apparaissait là magiquement sous nos yeux, tel une vision incarnée. Dans Signes de vie, tourné au même moment, Herzog organise une autre apparition : Strozeck parcourt les rues, une musique au piano se fait de plus en plus présente, on pense d'abord que c'est une musique mentale qui monte en lui, ainsi que donnent à le penser des plans de plus en plus rapprochés sur son visage, jusqu'à ce qu'il entrouvre une porte qui révèle un pianiste, qui n'est autre que Florian Fricke répétant une pièce virtuose. Là aussi, il s'agit pleinement de l'apparition d'un corps incarné : chemise ouverte qui donne à voir la peau sous le vêtement ; et détail qui a son importance, Florian Fricke a déposé ses bottes de militaire à côté du piano, il joue pieds nus.

On peut dire que c'est aussi ce que produisent les trois documentaires musicaux placés en regard des films, ils incarnent la musique dans le corps des musiciens. Alors que Herzog révèle les coulisses de la création, comme il aime souvent à le faire dans ses films ${ }^{11}$, il traite la présentation de Ernst Reijseger dans Ode to the Dawn of Man comme une réincarnation christique : un plan dans l'église où a été enregistrée la

11 Voir La transformation du monde en musique (1996), sur les coulisses du Festival de Bayreuth. 
musique cadre le Christ en croix et ses deux vitraux latéraux symétriques ; alors que musique et voix se déclenchent, la caméra descend pour arriver à Ernst Reijseger la musique s'est faite corps. Pour être un peu dialectique, Herzog insiste cependant aussi sur l'immatérialité de la musique, comme le signifient un plan d'oscilloscope montrant les courbes sonores, ou un panoramique remontant vers l'orgue dont émane une musique, mais dont on ne voit rien de tangible. Un autre plan débute sur le Christ en croix, et c'est cette fois un panoramique horizontal qui va permettre de révéler l'organiste demeuré dissimulé jusqu'ici derrière son instrument, établissant un lien sensible entre présence immatérielle du Christ et son incarnation dans la musique à travers le corps du musicien. Pour aller au bout de la démonstration, Herzog prolonge le panoramique jusqu'à Ernst Reijseger, en duo avec l'organiste et cette fois au même niveau que lui au balcon de l'église. On découvre alors un Reijseger jouant pieds nus ${ }^{12}$ ! Cette mise au jour, cette révélation des pieds ne peut qu'en évoquer une autre, funèbre cette fois : les pieds nus de Kaspar qui dépassent d'un linge blanc à la morgue avant son autopsie, et qui viennent tout simplement signifier après la séquence de vision dans le désert, la mort du personnage. Pour boucler la boucle, les pieds de Kaspar entrent eux-mêmes dans une circulation iconologique avec ceux dépassant du linceul dans La lamentation sur le Christ mort de Mantegna.

La première partie de The Wild Blue Yonder, intitulée Requiem For A Dying Planet ${ }^{13}$, présente en seulement six plans, sur les notes de l'air "Dank sei dir, Herr » de Händel, un constat très explicite : deux plans sous-marins dans les fonds bleus de l'océan ; deux plans de présentation d'une remontée d'un plongeur immobilisé dans un scaphandrier, tel un Ecce homo ; et deux plans qui montrent l'un une jambe, l'autre le torse du scaphandre - disloqué et vidé de sa substance humaine. Soit une foudroyante histoire de l'humanité en six plans, des origines sous-marine au passage sur terre incarnée dans un corps, qui finit démembré et évanoui.

Si Gesualdo semble introuvable dans le portait que Herzog lui consacre, c'est qu'il est partout, au gré d'un véritable parcours figuratif : dans les récits faits de lui, factuels ou inventés ; dans sa musique qui est jouée ; mais aussi de manière extensive, comme pour Derniers mots, qui entretient le doute sur l'adéquation entre le récit et le corps proposé. Après que le musicologue «factuel » nous a présenté les dernières années de la vie de Gesualdo, pour finir par nous annoncer sa mort, on enchaîne sur la même personne dirigeant un madrigal, pour passer ensuite à un mouvement de caméra qui décrit une sculpture de mater dolorosa ${ }^{14}$ : un Christ mort gisant, ses proches autour de

12 Herzog dit dans le film que c'est lui qui lui a conseillé et demandé de jouer pieds nus. L'hypothèse la plus probable, au regard du jeu des (ré)incarnations chez Herzog, consiste à penser que le cinéaste cherche à retrouver un peu de la présence de Florian chez Ernst, tout comme il fait allusion dans Grizzly Man à une ressemblance entre Treadwell et Klaus Kinski.

13 C'est à la fois le titre du premier acte de The Wild Blue Yonder et de l'album de Ernst Reijseger : Requiem For A Dying Planet. Sounds For Two Films By Werner Herzog: The Wild Blue Yonder, The White Diamond (Reijseger 2006).

14 Pour Leçon de ténèbres, Herzog utilise sur des plans aériens de Bagdad un passage du Peer Gynt de Grieg, qui donne une forte tonalité mélancolique et grave à la scène, et prend tout son sens dans la suivante : les images télévisuelles des bombardements nocturnes de la capitale irakienne. Le passage en question s'intitule « La mort d'Åase », un andante doloroso. 
lui, dont la Vierge Marie éplorée. La mort de Gesulado est donc figurée rien moins que par la mort du Christ ! Tous les plans qui suivent prolongent cette invention figurative : Gesualdo est maintenant un enfant transformé en ange aux allures d'Icare à l'occasion d'une fête votive ; son inverse diabolique ; un mannequin de joute; et in fine un jeune homme. Pour retrouver Gesualdo, à partir d'une peinture montrée en début de film, et littéralement l'incarner dans le temps présent, Herzog recourt à l'une de ses stratégies actorales favorites : solliciter un jeune homme, ici en costume médiéval ; lui donner une ligne de texte à jouer avec un téléphone portable, afin d'accentuer les anachronismes et les circulations temporelles; s'approcher de lui en gros plan de manière à ce qu'il ne puisse pas échapper à la caméra ; le déstabiliser une fois son rôle joué (Herzog déclare lui avoir fait d'étranges grimaces) ; et laisser tourner la caméra pour enregistrer ce moment de surprise et de latence. Le départ d'un madrigal de Gesualdo, apposé à même ce visage sur lequel éclate une étincelle de vérité, achève d'incarner le compositeur de la Renaissance dans ce corps contemporain, enfin retrouvé au terme d'une odyssée figurative. Traversant le temps, dépassant la mort, Gesualdo est bien vivant et réincarné au temps présent.

Comme Pasolini et son Matthieu, comme Rossellini et son Francesco, Herzog cherche le corps qui pourra incarner le mythe. Pour Grizzly Man, Herzog rassemble dans un avion puis sur le lieu de la mort de Treadwell trois de ses proches, que nous avions vu témoigner jusqu'ici séparément - déjà le remembrement d'une dispersion. Une musique de Richard Thompson et de son groupe éphémère se déclenche. Or la ligne mélodique de la guitare rappelle lointainement une chanson : Death Is Not the End de Bob Dylan. Et ainsi, quand Jewel, l'ex-compagne de Timothy, au moment de la dispersion des cendres, déclare à propos de Timothy : «Tu as réussi à vivre ici à jamais ", résonnent les paroles de Dylan : "Oh, the tree of life is growing / Where the spirit never dies ". Les plans aériens qui suivent ne font que confirmer la non-mort de Treadwell, passé par une sorte de palingénésie, une renaissance de la nature, dans les moindres fragments du parc national de Katmai. Le film accomplit l'un des rêves de Timothy, fuir la civilisation humaine et faire corps avec la nature ${ }^{15}$.

En conclusion du film, après s'être approché au plus près de la mort de Timothy en montrant les toutes dernières séquences qu'il a filmées, on revoit le personnage bien vivant et heureux marchant sous le soleil en compagnie de ses amis renards, une véritable vision du paradis sur terre. Un raccord sur le cockpit de l'avion nous fait directement passer au ciel. Le pilote et ami de Timothy chante par-dessus la chanson de clôture du film, une reprise d'un standard country : "Coyotes " de Bob McDill, interprétée par Don Edwards. Une chanson qui tombe à point, puisqu'elle parle du vieil Ouest disparu sous l'asphalte, l'acier et les puits de pétrole, et d'un cow-boy qui fuit cette civilisation galopante pour vivre avec les derniers animaux sauvages, eux

15 Tout comme en rêvait également le naturaliste John Muir : « Nous sommes dans la montagne, et la montagne est en nous, dans chacun de nos nerfs, pénétrant par chacun de nos pores, et notre corps, alors, devient transparent comme du verre à la beauté qui l'environne, comme s'il en était devenu une partie, vibrant avec l'air et les arbres, les courants et les rochers, dans les vagues du soleil - une partie de la nature, non plus vieille ou jeune, saine ou malade, mais immortelle » (Muir dans Le Bris [1992] 2009, p. 8-9). 
aussi menacés. Quand le pilote complète l'un des termes de la chanson, "And the lion is gone / And the red wolf is gone " par "And Treadwell is gone... ", il accomplit le mythe en faisant entrer Treadwell dans la culture populaire. Si l'on va maintenant au bout des paroles de la chanson, celle-ci raconte qu'un jour le cow-boy finit par disparaître, personne ne retrouve sa trace, mais le soir qui suit, on a entendu chanter un coyote de plus ${ }^{16}$. En poussant la logique de réincarnation plus loin encore, Herzog va même suggérer que s'il a reconnu quelque chose de lui-même chez Treadwell, c'est peut-être qu'une part de Treadwell est aussi en Herzog. La preuve, les choix musicaux que fait Herzog pour la création de Grizzly Man, d'après lui, Treadwell les aurait fait aussi : "C'est un grand cinéaste, je me suis demandé quel genre de musique il aurait choisi. Je pense qu'il se serait retrouvé dans ce studio avec Richard Thompson, avec toi et avec tous les autres »(Herzog 2005a). Aussi farfelue qu'elle apparaisse, cette hypothèse est encore renforcée, juste après cette déclaration, par un raccord entre un plan sur Herzog et un plan sur Timothy.

Ode to the Dawn of Man, enfin, constitue un précieux addendum à La grotte des rêves perdus. Tandis que La grotte des rêves perdus s'interroge sur le berceau de l'humanité et sur la naissance de l'homme comme artiste, de l'homme comme créateur ${ }^{17}$, le documentaire sur la création de sa musique, après avoir travaillé l'incarnation de la musique dans le corps du musicien, développe un parallèle entre musique, création et procréation. Herzog intègre à son film plusieurs scènes qui montrent le très jeune enfant de Reijseger et sa femme. D'abord dans une sorte de dialogue musical entre le père au violoncelle et les réactions de l'enfant, puis avec sa mère au piano, les petites mains posées sur les grandes. Le film s'est ouvert et se referme sur une bible luthérienne, elle-même ouverte puis refermée par Werner Herzog en personne. Une musique, un film et un enfant sont nés, comme l'aube d'une humanité nouvelle.

\section{Au CEEUR DU MONDE}

Les quatre pages de L'Image-mouvement que Gilles Deleuze consacre à Herzog restent sans doute aujourd'hui les plus fines et justes si l'on veut atteindre le cœur de l'œuvre (Deleuze 1983, p. 250-253). On y trouve notamment l'idée, à partir de l'analyse de Fitzcarraldo, d'un opéra de la nature, ou de la nature comme opéra. Dans La grotte des rêves perdus, Herzog fait ce commentaire sur des images de Vallon Pont d'Arc, le site des gorges de l'Ardèche où se situe la grotte :

\footnotetext{
16 "He disappeared without even a word / But that night as the moon crossed the mountain / One more coyote was heard».

17 Et comment ne pas penser ici à Georges Bataille : «Autrefois, la véritable naissance de l'art, l'époque à laquelle il avait pris le sens d'une éclosion miraculeuse de l'être humain, semblait beaucoup plus proche de nous. L'on parlait de miracle grec et c'était à partir de la Grèce que l'homme nous paraissait pleinement notre semblable. J'ai voulu souligner le fait que le moment de l'histoire le plus exactement miraculeux, le moment décisif, devait être reculé bien plus haut. Ce qui différencia l'homme de la bête a pris en effet pour nous la forme spectaculaire d'un miracle, mais ce n'est pas tellement du miracle grec que nous devrions désormais parler que du miracle de Lascaux» (Bataille [1955] 1979, p. 9).
} 
Il y a une aura mélodramatique dans ce paysage, qui pourrait être tout droit sorti d'un opéra de Wagner ou d'une peinture du Romantisme allemand. Est-ce là notre lien avec eux ? La mise en scène (staging) de ce paysage en tant qu'événement opératique n'appartient pas uniquement aux Romantiques. Les hommes de l'âge de pierre ont pu avoir un même sentiment de paysage intérieur (Herzog 2010).

Quand la musique imite le paysage et la nature, elle parviendrait donc à nous livrer un portrait intime de l'homme : il suffit d'écouter Chopin pour comprendre qu'il était méchant ; ou d'écouter la partition de Richard Thompson pour ressentir dans notre chair les fissures psychologiques de Treadwell.

Comme Marcel Conche établissait que Nature et mort ne formaient qu'une seule et même évidence, c'est l'opéra et la mort qui sont tout autant intimement liés. Ce dont on peut avoir le sentiment à l'aune de tous les films de Herzog, qui déploie d'infinies visions de la mort, et que son auteur confirme lui-même : "Opera's greatest theme - that's what I'm calling it, anyway - is death. It defines the outermost limit of what is even conceivable. Doesn't that link opera and cinema?" (Herzog [1993] 2014a, p. 123). Le plus grand opéra, c'est-à-dire la plus grande œuvre, c'est la mort. Qui est aussi une question essentielle pour la représentation et pour le cinéma. En matière de structure, de forme, un film comme Grizzly Man pourrait dès lors être envisagé comme une succession d'airs, de récitatifs, à une ou plusieurs voix, alternant moments musicaux intimistes ou symphoniques, figures statiques ou en mouvement sur la scène de la nature, soit un film-opéra habité par la question de la mort et des limites de sa représentation.

L'énigme de Kaspar Hauser s'ouvre et se termine sur un air de Tamino extrait de La flûte enchantée de Mozart. En ouverture, le rythme des rames d'une barque naviguant sur un lac s'agence parfaitement à celui de l'orchestre et de la voix du ténor qui s'élève, s'émerveillant à la découverte du portrait de Pamina : "Ce portrait est un ravissement / comme nul n'en vit jamais de pareil ! / Je le sens, devant cette image divine, / mon cœur connaît une émotion nouvelle. » Le naturaliste John Muir s'émerveillait devant les beautés de la nature comme chant et langage, source d'une pareille émotion, qui touche au cœur et en cela donne accès au cœur du monde :

Aussi longtemps que je vivrai, j'entendrai les chutes d'eau, le chant des oiseaux et du vent, j'apprendrai le langage des roches, le grondement des orages et des avalanches. Je me lierai aux glaciers et aux fleurs sauvages, et je resterai aussi près que possible du cour du monde (Muir dans Le Bris [1992] 2009, p. 11-12).

Werner Herzog, à l'écoute des volcans comme de nos fréquences émotionnelles intimes, se fait cinéaste-sismographe de notre expérience musicale du monde ${ }^{18}$.

Transformer le monde en musique, c'est s'interroger sur la Nature musicienne et sur l'homme qui s'efforce à son tour, comme en réponse, d'être musicien, jusque dans la dissonance. Kaspar l'exprime à sa manière, désespéré : "La musique se ressent fort dans ma poitrine ! Pourquoi ne joue-t-on pas du piano comme on respire ${ }^{19}$ ?»

18 Je partage cette idée, dans le cinéma de Herzog, du paysage comme électrocardiogramme ou relevé sismographique, avec Ames 2012.

19 "Die Musik fühlt mir stark in der Brust! Warum kann man das Klavier nicht spielen sowie das Atmen?». 
Or, quand il joue une valse de Mozart et qu'elle sonne comme le piano préparé de Jim O'Rourke, c'est sans doute à ce moment-là qu'il est au plus proche du cœur du monde. Face au modèle civilisationnel qu'on lui présente comme seul à imiter et à singer - la haute société dans sa plus grande facticité est associée à un quatuor à cordes -, Kaspar oppose son jeu de piano déstructuré, qu'il finira par interrompre, suffoquant, laissant planer un silence éloquent. Le film commençait sur cet incipit : "N'entendez-vous pas ces cris effroyables tout alentour, que communément on appelle le silence ? $^{20}$ ". Dans La grotte des rêves perdus, le chercheur Jean Clottes invitait lui aussi à sonder la profondeur abyssale du silence : "Écoutons le silence de la grotte, et peut-être entendrons nous le battement de nos cours » (Herzog 2010).

\section{BIBLIOGRAPHIE}

Ames, Eric (2012), Ferocious Reality. Documentary according to Werner Herzog, Minneapolis, University of Minnesota Press, 2012.

Bataille, Georges ([1955] 1979), Lascaux ou la naissance de l'art, dans Euvres complètes, vol. IX, Paris, Gallimard.

Blumenberg, Hans ([1981] 2010), L'imitation de la nature et autres essais esthétiques, traduit de l'allemand par Isabelle Kalinowski et Marc de Launay, Paris, Hermann Éditeurs.

Büchner, Georg ([1835] 2014), Lenz, traduit de l'allemand par Henri-Alexis Baatsch, Paris, Christian Bourgois éditeur.

Conche, Marcel (2001), Présence de la nature, Paris, PUF.

Daney, Serge (1981), « Le cru et le cuit », Cahiers du cinéma, n 323-324 (mai), p. 10-14.

Deleuze, Gilles (1983), Cinéma 1. L'Image-mouvement, Paris, Les Éditions de Minuit.

Eisenstein, Sergueï M. ([1945] 1952), « Naissance d'un film », Cahiers du cinéma, no 11 (avril), p. 1829.

Ginzburg, Carlo ([1998] 2001), À distance. Neuf essais sur le point de vue en histoire, traduit de l'italien par Pierre-Antoine Fabre, Paris, Gallimard.

Guido, Laurent (2013), «Dans les "abysses du temps”. Échos wagnériens dans l'œuvre documentaire de Werner Herzog ", Décadrages, no 25 (automne), p. 37-60.

Herzog, Werner (1974), Kaspar Hauser, Allemagne, 110 min.

Herzog, Werner (1975), « Entretien avec Werner Herzog par Michel Ciment », Positif, no 169 (mai), p. 6-15.

Herzog, Werner (2005a), In the Edges. The Grizzly Man Sessions, bonus du DVD Grizzly Man, Metropolitan Vidéo.

Herzog, Werner (2005b), Requiem in Space. Werner and Ernst Make Music, bonus du DVD The Wild Blue Yonder, Soda Pictures.

Herzog, Werner (2005c), Grizzly Man, États-Unis, 104 min.

Herzog, Werner (2008a), Conquête de l'inutile, Nantes, Capricci.

20 « N'entendez vous donc rien? N'entendez-vous donc pas cette voix épouvantable qui hurle de tout l'horizon et qu'on appelle d'ordinaire le silence? »(Büchner [1835] 2014, p. 71). 
Herzog, Werner (2008b), Manuel de survie. Entretien avec Hervé Aubron et Emmanuel Burdeau, Nantes, Capricci.

Herzog, Werner ([1985] 2014a), « David Knaus and Beat Presser. Discussion with Werner Herzog on Staging His First Opera », dans Eric Ames (éd.), Werner Herzog. Interviews, Jackson, University Press of Mississippi, p. 92-105.

Herzog, Werner ([1993] 2014a), " Alexander Schwarz. Interview with Werner Herzog on Lessons of Darkness", dans Eric Ames (éd.), Werner Herzog. Interviews, Jackson, University Press of Mississippi, p. 109-130.

Herzog, Werner ([2005] 2014a), « Cynthia Fuchs. A More Athletic Approach : An Interview with Werner Herzog on Grizzly Man ", dans Eric Ames (éd.), Werner Herzog. Interviews, Jackson, University Press of Mississippi, p. 155-159.

Herzog, Werner (2010), La grotte des rêves perdus, France/Allemagne, 90 min.

Herzog, Werner (2014b), A Guide to the Perplexed. Conversations with Paul Cronin, London, Faber \& Faber.

Le Bris, Michel ([1992] 2009), "Le chant du monde ", préface à John Muir, Voyages en Alaska (1913-1914), traduit de l'anglais par Jean-Yves Prate et Michel Le Bris, Paris, Payot/Voyageurs.

Lévi-Strauss, Claude (1964), Mythologiques 1. Le Cru et le Cuit, Paris, Plon.

Novalis ([1802] 1975), Les disciples à Saïs, dans Euvres complètes I, traduit de l'allemand par Armand Guerne, Paris, Gallimard.

Prager, Brad (2007), The Cinema of Werner Herzog. Aesthetic Ecstasy and Truth, London/New York, Wallflower.

Rogers, Holly (2012), " Death for Five Voices. Gesualdo's "Poetic truth" ", dans Brad Prager (dir.), A Companion to Werner Herzog, Oxford, Wiley-Blackwell, p. 187-207.

Reibel, Emmanuel (2016), La nature et la musique, Paris, Fayard.

Reijseger, Ernst (2006), Requiem For A Dying Planet. Sounds For Two Films By Werner Herzog: The Wild Blue Yonder, The White Diamond, Winter \& Winter, 910127. 